\title{
On decomposing graphs of large minimum degree into locally irregular subgraphs
}

\author{
Jakub Przybyło* \\ AGH University of Science and Technology \\ al. A. Mickiewicza 30 \\ 30-059 Krakow, Poland \\ jakubprz@agh.edu.pl
}

Submitted: Apr 8, 2015; Accepted: Apr 25, 2016; Published: May 13, 2016

Mathematics Subject Classifications: 05C78, 05C15

\begin{abstract}
A locally irregular graph is a graph whose adjacent vertices have distinct degrees. We say that a graph $G$ can be decomposed into $k$ locally irregular subgraphs if its edge set may be partitioned into $k$ subsets each of which induces a locally irregular subgraph in $G$. It has been conjectured that apart from the family of exceptions which admit no such decompositions, i.e., odd paths, odd cycles and a special class of graphs of maximum degree 3, every connected graph can be decomposed into 3 locally irregular subgraphs. Using a combination of a probabilistic approach and some known theorems on degree constrained subgraphs of a given graph, we prove this to hold for graphs of minimum degree at least $10^{10}$. This problem is strongly related to edge colourings distinguishing neighbours by the pallets of their incident colours and to the 1-2-3 Conjecture. In particular, the contribution of this paper constitutes a strengthening of a result of Addario-Berry, Aldred, Dalal and Reed [J. Combin. Theory Ser. B 94 (2005) 237-244].
\end{abstract}

Keywords: locally irregular graph; graph decomposition; edge set partition; 1-2-3 Conjecture

\section{Introduction}

All graphs considered are simple and finite. We follow [6] for the notations and terminology not defined here. A locally irregular graph is a graph where every vertex has degree distinct from the degrees of all of its neighbours. In other words, it is a graph

\footnotetext{
${ }^{*}$ Supported by the National Science Centre, Poland, grant no. 2014/13/B/ST1/01855 and partly supported by the Polish Ministry of Science and Higher Education.
} 
in which adjacent vertices have distinct degrees. Motivated by a few well known problems in edge colourings and labellings, we investigate a (non-evidently) related concept of decompositions of graphs into locally irregular subgraphs. More precisely, we say that a graph $G=(V, E)$ can be decomposed into $k$ locally irregular subgraphs if its edge set can be partitioned into $k$ subsets each of which induces a locally irregular subgraph, i.e., $E=E_{1} \cup E_{2} \cup \ldots \cup E_{k}$ with $E_{i} \cap E_{j}=\emptyset$ for $i \neq j$ and $H_{i}:=\left(V, E_{i}\right)$ is locally irregular for $i=1,2, \ldots, k$. Naturally, instead of decomposing the graph $G$, we may paint its edges with $k$ colours, say $1,2, \ldots, k$, so that every colour class induces a locally irregular subgraph in $G$. Such a colouring is called a locally irregular $k$-edge colouring of $G$. Equivalently it is just an edge colouring such that if an edge $u v \in E$ has colour $i \in\{1,2, \ldots, k\}$ assigned to it, then the number of edges coloured with $i$ incident with $u$ must be distinct from the number of edges coloured with $i$ incident with $v$. As a weakening of this property, we may require $u$ and $v$ to differ in frequencies of any of the colours from $\{1,2, \ldots, k\}$, not specifically the colour $i$ (which is assigned to $u v$ ). In other words, we want adjacent vertices to have distinct multisets of their incident colours under a colouring $c: E \rightarrow\{1,2, \ldots, k\}$. We call such a colouring a neighbour multiset distinguishing $k$-edge colouring. This variant of edge colourings has in fact already been investigated by Addario-Berry et al. in [2]. They proved that 4 colours are always sufficient to construct such a colouring for every graph containing no isolated edges, and provided the following improvement.

Theorem 1. There exists a neighbour multiset distinguishing 3-edge colouring of every graph $G$ of minimum degree at least $10^{3}$.

Their research was motivated by the so called 1-2-3 Conjecture due to Karoński, Łuczak and Thomason [8], yet another concept introducing 'local irregularity' into a graph. Let $c: E \rightarrow\{1,2, \ldots, k\}$ be an edge colouring of $G$ with positive integers. For every vertex $v$ we denote by $s_{c}(v):=\sum_{u \in N(v)} c(u v)$ the sum of its incident colours and call it the weighted degree of $v$. We say that $c$ is a neighbour sum distinguishing $k$-edge colouring of $G$ if $s_{c}(u) \neq s_{c}(v)$ for all adjacent vertices $u, v$ in $G$. Equivalently, instead of assigning integers from $\{1,2, \ldots, k\}$ to the edges, one might strive to multiply them the corresponding numbers of times in order to create a locally irregular multigraph of $G$, i.e., a multigraph in which adjacent vertices have distinct degrees. In [8] Karoński et al. posed the following elegant problem.

Conjecture 2 (1-2-3 Conjecture). There exists a neighbour sum distinguishing 3-edge colouring of every graph $G$ containing no isolated edges.

Thus far it is known that a neighbour sum distinguishing 5-edge colouring exists for every graph without isolated edges, see [7]. In fact our interest in locally irregular graphs originated from the 1-2-3 Conjecture via the following easy observation from [5].

Observation 3. If $G$ is a regular graph, then there exists a neighbour sum distinguishing 2-edge colouring of $G$ if and only if it can be decomposed into 2 locally irregular subgraphs. 
It is worth noting in this context that asymptotically almost surely a random $d$-regular graph can be decomposed into 2 locally irregular subgraphs for every constant $d>d_{0}$, where $d_{0}$ is a large constant, see [5]. In the same paper the authors investigate a special family $\mathfrak{T}$ of graphs of maximum degree (at most) 3 whose every member can be constructed from a triangle by repeatedly performing the following operation: choose a triangle with a vertex of degree 2 in our constructed graph and append to this vertex either a hanging path of even length or a hanging path of odd length with a triangle glued to its other end. They posed the following conjecture.

Conjecture 4. Every connected graph $G$ which does not belong to $\mathfrak{T}$ and is not an odd length path nor an odd length cycle can be decomposed into 3 locally irregular subgraphs.

The graphs excluded in the conjecture above were also proven to be the only connected graphs which do not admit decompositions into any number of locally irregular subgraphs. This conjecture was verified in [5] for some classes of graph, e.g., complete graph, complete bipartite graphs, trees, cartesian products of graphs with the desired property (hypercubes for instance), and for regular graphs with large degrees.

The main result of this paper is the following strengthening of Theorem 1, which confirms Conjecture 4 for graphs of sufficiently large minimum degree.

Theorem 5. Every graph $G$ with minimum degree at least $10^{10}$ can be decomposed into three locally irregular subgraphs.

Its proof combines a probabilistic approach with some known theorems on degree constrained subgraphs.

To exemplify the fact that the two graph invariants representing the minimum numbers of colors necessary to create a neighbour multiset distinguishing edge colouring and a locally irregular edge colouring, resp., are indeed distinct, let us consider a graph constructed as follows. Take a single edge, say $u v$, and append two hanging paths of length 2 to the vertex $u$ and another two hanging paths of length 2 to the vertex $v$. It is easy to see that there exist multiset distinguishing 2-edge colourings of this graph, but no locally irregular 2-edge colouring. This example may also be easily generalized by substituting the paths of length 2 with any other even paths.

In the following, given two graphs $H_{1}=\left(V_{1}, E_{1}\right), H_{2}=\left(V_{2}, E_{2}\right)$, usually subgraphs of a host graph $G$, by $H_{1} \cup H_{2}$ we shall mean the graph $\left(V_{1} \cup V_{2}, E_{1} \cup E_{2}\right)$. Moreover, we shall write $H_{2} \subset H_{1}$ if $V_{2} \subset V_{1}$ and $E_{2} \subset E_{1}$, and in case of $H_{2} \subset H_{1}$, we shall also write $H_{1}-E\left(H_{2}\right)$ to denote the graph obtained from $H_{1}$ by removing the edges of $H_{2}$. Given a subset $E^{\prime}$ of edges of a graph $G=(V, E)$, the graph induced by $E^{\prime}$ shall be understood as $G^{\prime}:=\left(V, E^{\prime}\right)$.

\section{Tools}

We shall use the Lovász Local Lemma and the Chernoff Bound, classical tools of the probabilistic method, see e.g. [4] and [9], respectively. 
Theorem 6 (The Local Lemma; General Case). Let $\mathcal{A}$ be a finite family of (typically bad) events in any probability space and let $D=(\mathcal{A}, E)$ be a directed graph such that every event $A \in \mathcal{A}$ is mutually independent of all the events $\{B:(A, B) \notin E\}$. Suppose that there are real numbers $x_{A}(A \in \mathcal{A})$ such that for every $A \in \mathcal{A}, 0 \leqslant x_{A}<1$ and

$$
\operatorname{Pr}(A) \leqslant x_{A} \prod_{B \leftarrow A}\left(1-x_{B}\right) .
$$

Then $\operatorname{Pr}\left(\bigcap_{A \in \mathcal{A}} \bar{A}\right)>0$.

Here $B \leftarrow A$ (or $A \rightarrow B$ ) means that there is an arc from $A$ to $B$ in $D$, the so called dependency digraph.

Theorem 7 (Chernoff Bound). For any $0 \leqslant t \leqslant n p$ :

$$
\operatorname{Pr}(|\operatorname{BIN}(n, p)-n p|>t)<2 e^{-\frac{t^{2}}{3 n p}},
$$

where $\operatorname{BIN}(n, p)$ is the sum of $n$ independent variables, each equal to 1 with probability $p$ and 0 otherwise.

For the deterministic part of our proof we shall in turn use a consequence of the following theorem from [1] (see also [2,3] for similar degree theorems and their applications).

Theorem 8. Suppose that for some graph $G=(V, E)$ we have chosen, for every vertex $v$, two integers:

$$
a_{v}^{-} \in\left[\frac{d(v)}{3}-1, \frac{d(v)}{2}\right], \quad a_{v}^{+} \in\left[\frac{d(v)}{2}-1, \frac{2 d(v)}{3}\right] .
$$

Then there exists a spanning subgraph $H$ of $G$ such that for every $v \in V$ :

$$
d_{H}(v) \in\left\{a_{v}^{-}, a_{v}^{-}+1, a_{v}^{+}, a_{v}^{+}+1\right\} .
$$

Corollary 9. Suppose that for some graph $G=(V, E)$ with minimum degree at least 12 we have chosen, for every vertex $v$, an integer $\lambda_{v} \geqslant 2$ with $6 \lambda_{v} \leqslant d(v)$. Then for every assignment

$$
t: V \rightarrow \mathbb{Z}
$$

there exists a spanning subgraph $H$ of $G$ such that $d_{H}(v) \in\left[\frac{d(v)}{3}, \frac{2 d(v)}{3}\right]$ and $d_{H}(v) \equiv t(v)$ $\left(\bmod \lambda_{v}\right)$ or $d_{H}(v) \equiv t(v)+1\left(\bmod \lambda_{v}\right)$ for each $v \in V$.

Proof. For every vertex $v \in V$ we have:

$$
\left\lfloor\frac{d(v)}{2}\right\rfloor-\left\lfloor\frac{d(v)}{3}\right\rfloor+1 \geqslant \frac{d(v)-1}{2}-\frac{d(v)}{3}+1>\frac{d(v)}{6} \geqslant \lambda_{v} .
$$

Since both sides of the inequality above are integers, then in fact:

$$
\left\lfloor\frac{d(v)}{2}\right\rfloor-\left\lfloor\frac{d(v)}{3}\right\rfloor \geqslant \lambda_{v}
$$


Analogously,

$$
\left\lfloor\frac{2 d(v)}{3}\right\rfloor-\left\lfloor\frac{d(v)}{2}\right\rfloor+1 \geqslant \frac{2 d(v)-2}{3}-\frac{d(v)}{2}+1>\frac{d(v)}{6} \geqslant \lambda_{v},
$$

hence,

$$
\left\lfloor\frac{2 d(v)}{3}\right\rfloor-\left\lfloor\frac{d(v)}{2}\right\rfloor \geqslant \lambda_{v}
$$

Thus the sets of integers

$$
\left\{\left\lfloor\frac{d(v)}{3}\right\rfloor+1, \ldots,\left\lfloor\frac{d(v)}{2}\right\rfloor\right\} \text { and }\left\{\left\lfloor\frac{d(v)}{2}\right\rfloor, \ldots,\left\lfloor\frac{2 d(v)}{3}\right\rfloor-1\right\}
$$

both contain all remainders modulo $\lambda_{v}$. The thesis follows then by Theorem 8 (it is sufficient to choose $a_{v}^{-}, a_{v}^{+}$from these sets, resp., so that $\left.a_{v}^{-}, a_{v}^{+} \equiv t(v)\left(\bmod \lambda_{v}\right)\right)$.

\section{Proof of Theorem 5}

\subsection{Notions}

Let $G=(V, E)$ be a graph of minimum degree at least $10^{10}$. In the following by $d(v)$ we shall mean the degree of a vertex $v$ in $G$, and we shall write $d(v)^{p}$ for short instead of $(d(v))^{p}$. Let us denote the auxiliary 'optimizing' constant

$$
\beta:=2^{\frac{1}{0.38}}
$$

where $\beta \approx 6.2(6.19<\beta<6.2)$, and set

$$
\alpha(v):=\left\lceil\log _{\beta} d(v)\right\rceil
$$

for every $v \in V$. In order to apply Corollary 9, we shall also need two auxiliary vertex labellings, say $c_{1}$ and $c_{2}$, with certain regular features. Thus for every vertex $v$ let us first randomly and independently choose one value in $\left\{0,1, \ldots, 2^{\alpha(v)}-1\right\}$, each with equal probability, and denote it by $c_{1}(v)$. Then let us independently repeat our drawing, i.e., again for every $v \in V$ randomly and independently we choose one value in $\left\{0,1, \ldots, 2^{\alpha(v)}-\right.$ $1\}$, each with equal probability, and denote it by $c_{2}(v)$.

By our construction below it shall be clear that every edge whose one end has the degree at least $\beta$ times bigger than the other will be 'safe' from any potential conflicts between its end-vertices. Some of the remaining edges shall require extra attention though, and shall thus be called 'risky'. We distinguish three kinds of these, i.e., we say that an edge $u v$ with $d(v) / \beta<d(u)<\beta d(v)$ (hence $\alpha(u)$ and $\alpha(v)$ differ by at most 1 ) is:

- risky of type 1 if $2^{\alpha(u)} c_{1}(u) \equiv 2^{\alpha(v)} c_{1}(v)\left(\bmod \min \left\{4^{\alpha(u)}, 4^{\alpha(v)}\right\}\right) ;$

- risky of type 2 if $2^{\alpha(u)} c_{2}(u) \equiv 2^{\alpha(v)} c_{2}(v)\left(\bmod \min \left\{4^{\alpha(u)}, 4^{\alpha(v)}\right\}\right) ;$ 
- risky of type 3 if

$\left|d(u)-3 \cdot 2^{\alpha(u)}\left(c_{1}(u)+c_{2}(u)\right)-d(v)+3 \cdot 2^{\alpha(v)}\left(c_{1}(v)+c_{2}(v)\right)\right|$

$<\min \left\{3 \cdot 2^{\alpha(u)}, 3 \cdot 2^{\alpha(v)}\right\}\left(\bmod \min \left\{3 \cdot 4^{\alpha(u)}, 3 \cdot 4^{\alpha(v)}\right\}\right)$,

where, given integers $b$ and $k$ with $b \in\{1, \ldots, k\}$, by writing $|a|<b(\bmod k)$ we mean that $a$ is an integer which is congruent to one of the following: $-b+1,-b+2, \ldots, b-1$ modulo $k$. Denote the sets of risky edges of types 1,2 and 3 by $R_{1}, R_{2}$ and $R_{3}$, respectively. For each $v \in V$, let us also denote:

$$
\begin{aligned}
& A(v):=\left\{u \in N_{G}(v): u v \in R_{1}\right\} \\
& B(v):=\left\{u \in N_{G}(v): u v \in R_{2}\right\} \\
& C(v):=\left\{u \in N_{G}(v): u v \in R_{3}\right\} \\
& F(v):=B(v) \cap C(v)=\left\{u \in N_{G}(v): u v \in R_{2} \cap R_{3}\right\} .
\end{aligned}
$$

\subsection{Probabilistic Lemma}

Claim 10. With positive probability, for every vertex $v \in V$ :

$$
\begin{aligned}
|A(v)|,|B(v)|,|C(v)| & \leqslant 8 d(v)^{0.62} \text { and } \\
|F(v)| & \leqslant 12 d(v)^{0.24} .
\end{aligned}
$$

Proof. For every $v \in V$, let $X_{v}, Y_{v}, Z_{v}, T_{v}$ be the random variables of the cardinalities of the sets $A(v), B(v), C(v), F(v)$, resp., and let $A_{v}, B_{v}, C_{v}, F_{v}$ denote the events that $X_{v}>8 d(v)^{0.62}, Y_{v}>8 d(v)^{0.62}, Z_{v}>8 d(v)^{0.62}$ and $T_{v}>12 d(v)^{0.24}$, respectively. Consider a vertex $v \in V$, and let $u$ be any of its neighbours with $d(u) \in(d(v) / \beta, \beta d(v))$. Note that $\alpha(u) \in\{\alpha(v)-1, \alpha(v), \alpha(v)+1\}$. Then for arbitrarily fixed $c_{1}^{*}, c_{2}^{*} \in\left\{0,1, \ldots, 2^{\alpha(v)}-1\right\}$ and $c_{3}^{*} \in\left\{0,1, \ldots, 2^{\alpha(u)}-1\right\}$, in all cases:

$$
\begin{aligned}
\operatorname{Pr}\left(u \in A(v) \mid c_{1}(v)=c_{1}^{*}\right) & \leqslant \frac{1}{2^{\alpha(v)-1}} \leqslant \frac{1}{2^{\log _{\beta} d(v)-1}} \\
& =\frac{2}{d(v)^{\frac{1}{\log _{2} \beta}}}=\frac{2}{d(v)^{0.38}} .
\end{aligned}
$$

To see the first inequality above, note that for any fixed $c_{1}^{*}$, in the case when $\alpha(u) \leqslant \alpha(v)$, $u \in A(v)$ (i.e., $\left.2^{\alpha(u)} c_{1}(u) \equiv 2^{\alpha(v)} c_{1}^{*}\left(\bmod 4^{\alpha(u)}\right)\right)$ for exactly one out of $2^{\alpha(u)} \geqslant 2^{\alpha(v)-1}$ admissible choices for $c_{1}(u)$, while in the case when $\alpha(u)=\alpha(v)+1, u \in A(v)$ (i.e., $\left.2^{\alpha(v)} \cdot 2 c_{1}(u) \equiv 2^{\alpha(v)} c_{1}^{*}\left(\bmod 4^{\alpha(v)}\right)\right)$ either for 0 or for 4 (dependent on the parity of $c_{1}^{*}$ ) out of $2^{\alpha(u)}=2^{\alpha(v)+1}$ admissible choices for $c_{1}(u)$. Analogously:

$$
\begin{aligned}
\operatorname{Pr}\left(u \in B(v) \mid c_{2}(v)=c_{2}^{*}\right) & \leqslant \frac{1}{2^{\alpha(v)-1}} \leqslant \frac{1}{2^{\log _{\beta} d(v)-1}} \\
& =\frac{2}{d(v)^{\frac{1}{\log _{2} \beta}}}=\frac{2}{d(v)^{0.38}}, \\
\operatorname{Pr}\left(u \in C(v) \mid c_{1}(v)=c_{1}^{*} \wedge c_{2}(v)=c_{2}^{*} \wedge c_{2}(u)\right. & \left.=c_{3}^{*}\right) \leqslant \frac{2}{2^{\alpha(v)-1}} \leqslant \frac{4}{d(v)^{\frac{1}{\log _{2} \beta}}}=\frac{4}{d(v)^{\cdot 38}} .
\end{aligned}
$$


To see the first inequality in (6), it is in turn sufficient to notice that for any fixed integer $d^{*}$ (in particular $d^{*}=d(u)-3 \cdot 2^{\alpha(u)} c_{3}^{*}-d(v)+3 \cdot 2^{\alpha(v)}\left(c_{1}^{*}+c_{2}^{*}\right)$ ), in the case when $\alpha(u) \leqslant \alpha(v)$, we have $\left|d^{*}-3 \cdot 2^{\alpha(u)} c_{1}(u)\right|<3 \cdot 2^{\alpha(u)}\left(\bmod 3 \cdot 4^{\alpha(u)}\right)$ for at most 2 admissible choices for $c_{1}(u)$ (cf. the definition of $C(v)$ ), while in the case when $\alpha(u)=\alpha(v)+1$, we have $\left|d^{*}-3 \cdot 2^{\alpha(v)} \cdot 2 c_{1}(u)\right|<3 \cdot 2^{\alpha(v)}\left(\bmod 3 \cdot 4^{\alpha(v)}\right)$ for at most 4 admissible choices for $c_{1}(u)$.

Hence, by (6) and the law of total probability:

$$
\operatorname{Pr}\left(u \in C(v) \mid c_{1}(v)=c_{1}^{*} \wedge c_{2}(v)=c_{2}^{*}\right) \leqslant \frac{4}{d(v)^{0.38}} .
$$

Finally, since the choices for $c_{1}$ and $c_{2}$ are independent, by (5) and (6),

$$
\begin{aligned}
\operatorname{Pr}\left(u \in F(v) \mid c_{1}(v)=c_{1}^{*} \wedge c_{2}(v)=c_{2}^{*}\right)= & \operatorname{Pr}\left(u \in C(v) \mid c_{1}(v)=c_{1}^{*} \wedge c_{2}(v)=c_{2}^{*} \wedge u \in B(v)\right) \\
& \times \operatorname{Pr}\left(u \in B(v) \mid c_{1}(v)=c_{1}^{*} \wedge c_{2}(v)=c_{2}^{*}\right) \\
\leqslant & \frac{4}{d(v)^{0.38}} \cdot \frac{2}{d(v)^{0.38}}=\frac{8}{d(v)^{0.76}}
\end{aligned}
$$

Consequently, since all choices are independent and $2 / d(v)^{0.38} \leqslant 4 / d(v)^{0.38}$, by $(4)$ and the Chernoff Bound we obtain:

$$
\begin{aligned}
\operatorname{Pr}\left(A_{v} \mid c_{1}(v)=c_{1}^{*}\right) & =\operatorname{Pr}\left(X_{v}>8 d(v)^{0.62} \mid c_{1}(v)=c_{1}^{*}\right) \\
& \leqslant \operatorname{Pr}\left(\operatorname{BIN}\left(d(v), \frac{4}{d(v)^{0.38}}\right)>8 d(v)^{0.62}\right) \\
& \leqslant \operatorname{Pr}\left(\left|\operatorname{BIN}\left(d(v), \frac{4}{d(v)^{0.38}}\right)-4 d(v)^{0.62}\right|>4 d(v)^{0.62}\right) \\
& <2 e^{-\frac{4 d(v)^{0.62}}{3}} .
\end{aligned}
$$

By the law of total probability we thus obtain that:

$$
\operatorname{Pr}\left(A_{v}\right)<2 e^{-\frac{4 d(v)^{0.62}}{3}} .
$$

Analogously, by (5) and (7),

$$
\operatorname{Pr}\left(B_{v}\right)<2 e^{-\frac{4 d(v)^{0.62}}{3}} \text { and } \operatorname{Pr}\left(C_{v}\right)<2 e^{-\frac{4 d(v)^{0.62}}{3}} .
$$

Finally, again by the Chernoff Bound and (8):

$$
\begin{aligned}
\operatorname{Pr}\left(F_{v} \mid c_{1}(v)=c_{1}^{*} \wedge c_{2}(v)=c_{2}^{*}\right) & =\operatorname{Pr}\left(T_{v}>12 d(v)^{0.24} \mid c_{1}(v)=c_{1}^{*} \wedge c_{2}(v)=c_{2}^{*}\right) \\
& \leqslant \operatorname{Pr}\left(\operatorname{BIN}\left(d(v), \frac{8}{d(v)^{0.76}}\right)>12 d(v)^{0.24}\right) \\
& \leqslant \operatorname{Pr}\left(\left|\operatorname{BIN}\left(d(v), \frac{8}{d(v)^{0.76}}\right)-8 d(v)^{0.24}\right|>4 d(v)^{0.24}\right) \\
& <2 e^{-\frac{2 d(v)^{0.24}}{3}}
\end{aligned}
$$


and hence, by the law of total probability,

$$
\operatorname{Pr}\left(F_{v}\right)<2 e^{-\frac{2 d(v)^{0.24}}{3}} .
$$

Note that for every vertex $v \in V$, the events $A_{v}, B_{v}, C_{v}$ and $F_{v}$ depend only on the random choices for $v$ and its adjacent vertices $u$ with $d(v) / \beta<d(u)<\beta d(v)$. Thus each of these events (corresponding to the vertex $v$ ) is mutually independent of all events except (possibly) for these corresponding to the vertex $v$ itself, those corresponding to the neighbours $v^{\prime}$ of $v$ with $d(v) / \beta<d\left(v^{\prime}\right)<\beta d(v)$ and those corresponding to the neighbours $v^{\prime \prime}$ of such $v^{\prime}$ for which $d\left(v^{\prime}\right) / \beta<d\left(v^{\prime \prime}\right)<\beta d\left(v^{\prime}\right)$. In order to construct a dependency digraph $D$ necessary to apply Theorem 6 , from each of the events $A_{v}, B_{v}, C_{v}, F_{v}$, we draw arrows pointing at all other events corresponding to the vertices $w\left(w=v\right.$ or $w=v^{\prime}$ or $w=v^{\prime \prime}$ ) with the properties described above, for $v \in V$. For any event $L$ corresponding to a vertex $v$ of degree $d$ in $G$ (i.e., $L=A_{v}, L=B_{v}, L=C_{v}$ or $L=F_{v}$ ), we then set

$$
x_{L}=\frac{1}{1+d^{3}} .
$$

By our construction, for every such $L$,

$$
d_{D}^{+}(L) \leqslant 3+4 d+4 d(\lfloor\beta d\rfloor-1)=3+4 d\lfloor\beta d\rfloor,
$$

where $d_{D}^{+}(L)$ is the out-degree of $L$ in $D$. Moreover, if $L \rightarrow Q$ in $D$, then $Q$ is an event corresponding to a vertex $w$ with

$$
\frac{d}{\beta^{2}}<d(w)<\beta^{2} d
$$

By (12), (13) and (14), since $\frac{x}{1+x}>e^{-\frac{1}{x}}$ for $x>0$, we thus obtain:

$$
\begin{aligned}
x_{L} \prod_{Q \leftarrow L}\left(1-x_{Q}\right) & =\left[x_{L} \cdot \frac{1}{1-x_{L}}\right] \cdot\left[\left(1-x_{L}\right) \prod_{Q \leftarrow L}\left(1-x_{Q}\right)\right] \\
& >\left[\frac{1}{1+d^{3}} \cdot \frac{1}{1-\frac{1}{1+d^{3}}}\right]\left[\left(1-\frac{1}{1+\left(\frac{d}{\beta^{2}}\right)^{3}}\right)^{1+(3+4 d\lfloor\beta d\rfloor)}\right] \\
& \left.\geqslant \frac{1}{d^{3}}\left(\frac{\left(\frac{d}{\beta^{2}}\right)^{3}}{1+\left(\frac{d}{\beta^{2}}\right)^{3}}\right)^{25 d^{2}}>\frac{1}{d^{3}} e^{-\frac{1}{\left(\frac{d}{\beta^{2}}\right)^{3}} 25 d^{2}}\right] \\
& =\left(2 \frac{1}{2 d^{3}}\right)\left(e^{-\frac{25 \cdot \beta^{6}}{d}}\right)>\left(2 e^{-\frac{d^{0.24}}{3}}\right)\left(e^{-\frac{d^{0.24}}{3}}\right)=2 e^{-\frac{2 d^{0.24}}{3}}
\end{aligned}
$$

where $e^{-\frac{25 \cdot \beta^{6}}{d}} \geqslant e^{-\frac{d^{0.24}}{3}}$ for $d \geqslant\left(3 \cdot 25 \cdot \beta^{6}\right)^{\frac{1}{1.24}} \approx 221,460$, while $\frac{1}{2 d^{3}}>e^{-\frac{d^{0.24}}{3}}$ is equivalent to the inequality

$$
f(d):=\frac{d^{0.24}}{3}-\ln \left(2 d^{3}\right)>0
$$


which holds e.g. for $d \geqslant 10^{10}$, since $f\left(10^{10}\right) \approx 14>0$ and

$$
f^{\prime}(d)=\frac{0.08}{d^{0.76}}-\frac{3}{d}>0
$$

for $d>\left(\frac{3}{0.08}\right)^{\frac{1}{0.24}} \approx 3,617,959$.

By (9), (10), (11), (15) and the Local Lemma we thus obtain that

$$
\operatorname{Pr}\left(\bigcap_{v \in V} \overline{A_{v}} \cap \overline{B_{v}} \cap \overline{C_{v}} \cap \overline{F_{v}}\right)>0 .
$$

\subsection{Construction}

Suppose then that we have chosen the labellings $c_{1}$ and $c_{2}$ so that (2) and (3) hold for every $v \in V$. We shall use twice the fact that

$$
\frac{d}{3}-16 d^{0.62}>72 d^{0.76}
$$

for $d \geqslant 10^{10}$. Indeed, if $d>16^{\frac{1}{0.14}} \approx 398,893,555$, then the left hand side of inequality (16) is greater than $\frac{d}{3}-d^{0.76}$, what in turn is greater than the right hand side of inequality (16) if $d>(3 \cdot 73)^{\frac{1}{0.24}} \approx 5,647,425,084$.

Let us temporarily remove from $G$ all risky edges of type 1 and denote the graph obtained by $G^{\prime}$. By (2) and (16), for every vertex $v \in V$,

$$
\begin{aligned}
d_{G^{\prime}}(v) & \geqslant d(v)-8 d(v)^{0.62} \geqslant 72 \cdot d(v)^{0.76} \\
& =72 \cdot 4^{\log _{\beta} d(v)} \geqslant 6 \cdot 3 \cdot 4^{\alpha(v)} .
\end{aligned}
$$

By (17) and Corollary 9, we may thus find a subgraph $H_{1}$ of $G^{\prime}$ such that $d_{H_{1}}(v)$ has one of the two remainders modulo $3 \cdot 4^{\alpha(v)}$, namely:

$$
d_{H_{1}}(v) \equiv 3 \cdot 2^{\alpha(v)} c_{1}(v), 3 \cdot 2^{\alpha(v)} c_{1}(v)+1 \quad\left(\bmod 3 \cdot 4^{\alpha(v)}\right)
$$

for every $v \in V$, and (by (17)):

$$
d_{H_{1}}(v) \in\left[\frac{d_{G^{\prime}}(v)}{3}, \frac{2 d_{G^{\prime}}(v)}{3}\right] \subset\left[\frac{d(v)-8 d(v)^{0.62}}{3}, \frac{2 d(v)}{3}\right] .
$$

We paint the edges of $H_{1}$ with colour 1 , and claim that $H_{1}$ is locally irregular, what shall be exhibited in the next subsection.

Denote by $G_{1}$ the graph obtained from $G$ by removing all (already painted) edges of $H_{1}$. Let us (again temporarily) remove from $G_{1}$ all risky edges $e \in R_{2} \cup R_{3}$ of types 2 and 3 , and denote the graph obtained by $G^{\prime \prime}$. By (2), (16) and (19), for every vertex $v \in V$,

$$
\begin{aligned}
d_{G^{\prime \prime}}(v) & \geqslant \frac{d(v)}{3}-16 d(v)^{0.62} \geqslant 72 \cdot d(v)^{0.76} \\
& =72 \cdot 4^{\log _{\beta} d(v)} \geqslant 6 \cdot 3 \cdot 4^{\alpha(v)} .
\end{aligned}
$$


Let $C$ be the subgraph induced by the edges of $G_{1}$ which belong to $R_{3}$, hence $C$ and $G^{\prime \prime}$ are edge-disjoint. For every $v \in V$, denote by

$$
c_{v}:=d_{C}(v)=\left|C(v) \cap N_{G_{1}}(v)\right|
$$

the number of risky edges of type 3 incident with $v$ in $G_{1}$.

Subsequently consider the subgraph $F$ induced by these edges of $G_{1}$ which belong to $R_{2} \cap R_{3}$. Note that $F \subset C$. By (3), for every vertex $v \in V$,

$$
d_{F}(v) \leqslant 12 d(v)^{0.24}<\frac{1}{2} d(v)^{0.38},
$$

where the second inequality holds since $d(v)>24 \frac{1}{0.14} \approx 7,221,904,256$, and thus

$$
d_{F}(v)<\frac{1}{2} d(v)^{\frac{1}{\log _{2} \beta}}=2^{\log _{\beta} d(v)-1} \leqslant 2^{\alpha(v)-1} .
$$

Since both sides of the inequality above are integers, we in fact obtain that $d_{F}(v) \leqslant$ $2^{\alpha(v)-1}-1$. Hence, we may greedily find a proper vertex colouring

$$
h: V \rightarrow\left\{0,1, \ldots, 2^{\left\lceil\log _{\beta} \Delta(G)\right\rceil-1}-1\right\}
$$

of $F$ so that

$$
h(v) \leqslant 2^{\alpha(v)-1}-1
$$

for every $v \in V$.

By (20) and Corollary 9, we then may find a subgraph $H^{\prime \prime}$ of $G^{\prime \prime}$ such that

$$
\begin{aligned}
d_{H^{\prime \prime}}(v) \equiv & 3 \cdot 2^{\alpha(v)} c_{2}(v)+3 h(v)-c_{v} \\
& 3 \cdot 2^{\alpha(v)} c_{2}(v)+3 h(v)-c_{v}+1 \quad\left(\bmod 3 \cdot 4^{\alpha(v)}\right)
\end{aligned}
$$

for every $v \in V$, and (by (2))

$$
\begin{aligned}
d_{H^{\prime \prime}}(v) & \in\left[\frac{d_{G^{\prime \prime}}(v)}{3}, \frac{2 d_{G^{\prime \prime}}(v)}{3}\right] \\
& \subset\left[\frac{d(v)-d_{H_{1}}(v)-16 d(v)^{0.62}}{3}, \frac{2\left(d(v)-d_{H_{1}}(v)\right)}{3}\right] .
\end{aligned}
$$

Then we denote $H_{2}:=H^{\prime \prime} \cup C, H_{3}:=G-E\left(H_{1} \cup H_{2}\right)$, and colour the edges of the first of these graphs with 2 , while the edges of the second one with 3 . Note that since $H_{3}=G_{1}-E\left(H^{\prime \prime} \cup C\right)$, then $H_{3}$ contains no risky edges of type 3 .

\subsection{Validity}

Consider an edge $u v \in E\left(H_{1}\right)$. By our construction, $u v \notin R_{1}$. Condition (18) implies that either $d_{H_{1}}(u)$ or $d_{H_{1}}(u)-1$ must be a multiple of $\min \left\{3 \cdot 2^{\alpha(u)}, 3 \cdot 2^{\alpha(v)}\right\}$, and similarly, either $d_{H_{1}}(v)$ or $d_{H_{1}}(v)-1$ is a multiple of the same quantity. If $d(v) / \beta<d(u)<\beta d(v)$, 
then these multiples cannot be the same, since otherwise we would have $3 \cdot 2^{\alpha(u)} c_{1}(u)+$ $k_{u} \cdot 3 \cdot 4^{\alpha(u)}=3 \cdot 2^{\alpha(v)} c_{1}(v)+k_{v} \cdot 3 \cdot 4^{\alpha(v)}$ for some integers $k_{u}, k_{v}$, and hence $u v$ would have to be a risky edge of type 1 . Therefore, $d_{H_{1}}(u) \neq d_{H_{1}}(v)$ in this case. The same holds also for the (remaining) edges with a greater spread between the degrees of the end-vertices. We shall exhibit this explicitly for all such edges at the end of the proof.

Since $H^{\prime \prime}$ and $C$ are edge-disjoint, by (21) and (23),

$$
\begin{aligned}
d_{H_{2}}(v) \equiv & 3 \cdot 2^{\alpha(v)} c_{2}(v)+3 h(v), \\
& 3 \cdot 2^{\alpha(v)} c_{2}(v)+3 h(v)+1 \quad\left(\bmod 3 \cdot 4^{\alpha(v)}\right)
\end{aligned}
$$

for every $v \in V$. Consider an edge $u v \in E\left(H_{2}\right)$ with $d(v) / \beta<d(u)<\beta d(v)$. Then either $d_{H_{2}}(u)-3 h(u)$ or $d_{H_{2}}(u)-3 h(u)-1$ must be a multiple of $\min \left\{3 \cdot 2^{\alpha(u)}, 3 \cdot 2^{\alpha(v)}\right\} \geqslant$ $\max \left\{3 \cdot 2^{\alpha(u)-1}, 3 \cdot 2^{\alpha(v)-1}\right\}$, and similarly, either $d_{H_{2}}(v)-3 h(v)$ or $d_{H_{2}}(v)-3 h(v)-1$ is a multiple of the same quantity. If $u v \notin R_{2}$, then analogously as above these multiples cannot be the same, hence as by $(22), 0 \leqslant 3 h(u), 3 h(v) \leqslant \max \left\{3 \cdot 2^{\alpha(u)-1}, 3 \cdot 2^{\alpha(v)-1}\right\}-3$, we obtain that $d_{H_{2}}(u) \neq d_{H_{2}}(v)$. Otherwise, by our construction, $u v \in R_{2} \cap R_{3}$ is an edge of $F$, and hence $d_{H_{2}}(u) \neq d_{H_{2}}(v)$ by the 'properness' of $h$, since $0 \leqslant 3 h(u), 3 h(v) \leqslant$ $\max \left\{3 \cdot 2^{\alpha(u)-1}, 3 \cdot 2^{\alpha(v)-1}\right\}-3$.

By (18) and (25),

$$
\begin{aligned}
d_{H_{3}}(v) \equiv & d(v)-3 \cdot 2^{\alpha(v)}\left(c_{1}(v)+c_{2}(v)\right)-3 h(v) \\
& d(v)-3 \cdot 2^{\alpha(v)}\left(c_{1}(v)+c_{2}(v)\right)-3 h(v)-1 \\
& d(v)-3 \cdot 2^{\alpha(v)}\left(c_{1}(v)+c_{2}(v)\right)-3 h(v)-2 \quad\left(\bmod 3 \cdot 4^{\alpha(v)}\right)
\end{aligned}
$$

for every $v \in V$. Then, similarly as above, $d_{H_{3}}(u) \neq d_{H_{3}}(v)$ for every edge $u v$ of $H_{3}$ with $d(v) / \beta<d(u)<\beta d(v)$, since then $u v \notin R_{3}$ by our construction.

As for the remaining edges of $H_{1}, H_{2}$ and $H_{3}$, let us first note that for every vertex $v \in V$, by (19),

$$
d_{H_{1}}(v) \in\left[\frac{1}{3} d(v)-\frac{8}{3} d(v)^{0.62}, \frac{2}{3} d(v)\right],
$$

hence, by (2), (24), (27) and our construction,

$$
\begin{aligned}
d_{H_{2}}(v) \in & {\left[\frac{1}{3} d(v)-\frac{1}{3} d_{H_{1}}(v)-\frac{16}{3} d(v)^{0.62}, \frac{2}{3} d(v)-\frac{2}{3} d_{H_{1}}(v)+8 d(v)^{0.62}\right] } \\
\subset & {\left[\frac{1}{3} d(v)-\frac{1}{3} \cdot \frac{2}{3} d(v)-\frac{16}{3} d(v)^{0.62},\right.} \\
& \left.\frac{2}{3} d(v)-\frac{2}{3}\left(\frac{1}{3} d(v)-\frac{8}{3} d(v)^{0.62}\right)+8 d(v)^{0.62}\right] \\
= & {\left[\frac{1}{9} d(v)-\frac{16}{3} d(v)^{0.62}, \frac{4}{9} d(v)+\frac{88}{9} d(v)^{0.62}\right], }
\end{aligned}
$$


and thus, by (27) and (28),

$$
\begin{aligned}
d_{H_{3}}(v) \in & {\left[d(v)-d_{H_{1}}(v)-\left(\frac{2}{3} d(v)-\frac{2}{3} d_{H_{1}}(v)+8 d(v)^{0.62}\right),\right.} \\
& \left.d(v)-d_{H_{1}}(v)-\left(\frac{1}{3} d(v)-\frac{1}{3} d_{H_{1}}(v)-\frac{16}{3} d(v)^{0.62}\right)\right] \\
= & {\left[\frac{1}{3} d(v)-\frac{1}{3} d_{H_{1}}(v)-8 d(v)^{0.62}, \frac{2}{3} d(v)-\frac{2}{3} d_{H_{1}}(v)+\frac{16}{3} d(v)^{0.62}\right] } \\
\subset & {\left[\frac{1}{3} d(v)-\frac{1}{3} \cdot \frac{2}{3} d(v)-8 d(v)^{0.62},\right.} \\
& \left.\frac{2}{3} d(v)-\frac{2}{3}\left(\frac{1}{3} d(v)-\frac{8}{3} d(v)^{0.62}\right)+\frac{16}{3} d(v)^{0.62}\right] \\
= & {\left[\frac{1}{9} d(v)-8 d(v)^{0.62}, \frac{4}{9} d(v)+\frac{64}{9} d(v)^{0.62}\right] . }
\end{aligned}
$$

Consequently, since $\frac{4}{9} d+\frac{88}{9} d^{0.62} \leqslant \frac{2}{3} d$ for $d \geqslant 44 \frac{1}{0.38} \approx 21,129$, by (27), (29) and (30),

$$
d_{H_{1}}(v), d_{H_{2}}(v), d_{H_{3}}(v) \in\left[\frac{1}{9} d(v)-8 d(v)^{0.62}, \frac{2}{3} d(v)\right]
$$

and thus

$$
d_{H_{1}}(v), d_{H_{2}}(v), d_{H_{3}}(v) \in\left[\frac{4}{37} d(v), \frac{2}{3} d(v)\right],
$$

since $\frac{1}{9} d-8 d^{0.62} \geqslant \frac{4}{37} d$ for $d \geqslant(8 \cdot 333)^{\frac{1}{0.38}} \approx 1,034,102,857$. Since $\frac{2}{3} / \frac{4}{37}<6.17<\beta$, this guarantees that if (without the loss of generality) $d(u) \geqslant \beta d(v)$ for some edge $u v \in E$, then $d_{H_{1}}(u) \neq d_{H_{1}}(v), d_{H_{2}}(u) \neq d_{H_{2}}(v)$ and $d_{H_{3}}(u) \neq d_{H_{3}}(v)$. All subgraphs $H_{1}, H_{2}$ and $H_{3}$ are thus locally irregular.

\section{Concluding remarks}

Note that Conjecture 4 still remains open. It would be interesting to settle it at least for bipartite graphs.

Problem 11. Can every connected bipartite graph which is not an odd length path be decomposed into three locally irregular subgraphs?

Moreover, thus far it is not even known if any finite number of locally irregular subgraphs admitted is sufficient in general.

Problem 12. Does there exist a constant $K$ such that every connected graph which does not belong to $\mathfrak{T}$ and is not an odd length path nor an odd length cycle can be decomposed into (at most) $K$ locally irregular subgraphs?

This is not known in the case of bipartite graphs either. 


\section{References}

[1] L. Addario-Berry, K. Dalal, C. McDiarmid, B.A. Reed, A. Thomason. VertexColouring Edge-Weightings. Combinatorica, 27(1):1-12, 2007.

[2] L. Addario-Berry, R.E.L. Aldred, K. Dalal, B.A. Reed. Vertex colouring edge partitions. J. Combin. Theory Ser. B, 94(2):237-244, 2005.

[3] L. Addario-Berry, K. Dalal, B.A. Reed. Degree Constrained Subgraphs. Discrete Appl. Math., 156(7):1168-1174, 2008.

[4] N. Alon, J.H. Spencer. The Probabilistic Method, 2nd edition, Wiley, New York, 2000.

[5] O. Baudon, J. Bensmail, J. Przybyło, M. Woźniak. On decomposing regular graphs into locally irregular subgraphs. European J. Combin. 49:90-104, 2015.

[6] R. Diestel. Graph Theory, Electronic Edition 2005, Springer, New York, 2005.

[7] M. Kalkowski, M. Karoński, F. Pfender. Vertex-coloring edge-weightings: Towards the 1-2-3 conjecture. J. Combin. Theory Ser. B, 100:347-349, 2010.

[8] M. Karoński, T. Łuczak, A. Thomason. Edge weights and vertex colours. J. Combin. Theory Ser. B, 91:151-157, 2004.

[9] M. Molloy, B. Reed. Graph Colouring and the Probabilistic Method, Springer, Berlin, 2002. 